\title{
The Protocol for the Early vs. Late Infantile Strabismus Surgery Study
}

\author{
The Early vs. Late Infantile Strabismus Surgery Study Group*
}

(Comment: Below follows the complete text of the protocol of the multicentre study that will compare the results of early with those of late surgery in cases of infantile strabismus. At the last meeting of the E.S.A. in Brussels, the results of the pilot-study assessing the accuracy and reproducibility of orthoptic examination at age one were presented (also published in a previous issue of Strabismus). After that, the Study Group met and the decision was taken to proceed with the main study. Three committees were installed that would (1) set guidelines for surgical procedure and age limits for all surgeons operating early, (2) set guidelines for surgical procedures and age limits for all surgeons operating late and (3) set guidelines for amblyopia treatment for all participants in the study. Discussions about these three difficult subjects could not be carried out in detail during the meeting itself because it would certainly have taken several days to reach consensus among all participants.

Evidently, the committees have been trying to reach solutions that would be acceptable to all or most participants. This may, however, not be so in each individual case. We hope that not too many people will consider the guidelines as being incompatible with their participation in the study. Very difficult compromises were reached about leaving amblyopia in the study, and about permitting Faden surgery.

Initially Dr. Kolling, Dr. Haag and I had planned to start the trial on January 1st, but writing, translating, re-reading and correcting the protocol was a long and cumbersome job. Now we have started officially on May 1st. Anybody interested in participating in the study should contact the coordinator for the study in his country; the names and addresses are listed at the end of the protocol.

HuIB SIMONSz)

\begin{abstract}
The Early vs. Late Infantile Strabismus Surgery Study Group is a group of strabismologists and orthoptists who investigate whether early or late surgery is preferable in infantile strabismus, in a non-randomized, prospective, multi-centre trial. Infants between 6 and 18 months of age will receive a standardized entry examination and then be operated either before their second anniversary in clinics A, or between their 32nd and 60th month of age in clinics B. The children will be evaluated at age six. After completion of the study, the two groups can then be compared regarding degree of binocular vision, angle of strabismus and visual acuity of the worse eye relative to the better.
\end{abstract}

* The members of the Early vs. Late Infantile Strabismus Surgery Study Group were listed in Strabismus 1993; 1(2): $75-83$.

\section{INTRODUCTION}

Infantile strabismus starts before the age of six months. It is a convergent strabismus in most 
cases. The child concerned will fail to develop binocular vision or lose it rapidly. The child will suppress the image of one eye. Some children will alternate vision with either eye, others will use one eye preferentially, resulting in partial loss of central vision in that eye, amblyopia. Amblyopia is reversible by patching the healthy cye in most, but not in all cases. Infantile strabismus is very common: its incidence is about $1 \%$ in the general population. Strabismus operations for infantile strabismus are, hence, carried out in large numbers.

In the United States, surgery for infantile strabismus is now generally performed at an early age (age $1-2$ ), whereas in Europe many eye clinics still operate late (age 4-5). In Europe, children with infantile strabismus are treated differently depending on to which strabismologist or orthoptist they are referred.

The tendency to operate early developed in the nineteen-seventies. The main argument employed was that binocular vision can be restored if the child is operated early enough. However, to what extent binocular vision can be restored has not been measured in a controlled study. Most reports concerning this controversy so far were uncontrolled studies or clinical observations. ${ }^{1-9}$ One of the few controlled studies, a prospective assessment of acuity and stereopsis in amblyopic infantile esotropes following early surgery ${ }^{10}$ showed moderate degrees of stereopsis in $35 \%$ of the children who were operated early. In that study, three out of the series of 84 children had full stereopsis. These three did not need surgery, probably because they had intermittent strabismus.

The principal arguments of the proponents of early surgery are that:

- chances for the development of binocular vision are better,
- the parent-child relation is not disturbed by the cosmetic appearance of the child,

- the psychomotoric development of the child is better when the eyes are straight and

- an operation and a hospitalization at age 4-5 constitute a greater psychic trauma.

The principal arguments of the proponents of late surgery are that:

- the correction of the angle of strabismus can be more precise in surgery at age 4-5,

- secondary motility disorders (up- and downshoot in adduction, V- and A-pattern), can be corrected together with correction of the horizontal angle of strabismus in surgery at age $4-5$,

- the number of operations necessary in early surgery is larger because of the first two factors and

- treatment of amblyopia is more difficult after early surgery, because of:

- more difficult assessment by the treating orthoptist of alternating use of both eyes,

- difficulty in assessing visual acuity at age $0-2$,

- and lack of motivation among the parents of the child to patch one eye when their child has only a small, inconspicuous angle of strabismus.

Cosmetic considerations are also very important. It seems possible that, in this regard, there is a critical threshold in the swing towards early surgery: In a society where a very high percentage of the squinting children is operated upon at an early age, the remaining squinting children will become cosmetically less acceptable, and this may decide the balance in favour of early surgery in an entire country.

It will be very important for further developments in the field of strabismus to know what surgical therapy, at what age, is best for the 
child. It is likely that different aspects of therapeutic success, concerning the number of operations, the degree of binocular vision reached, the final angle of strabismus and the degree of success in the treatment of the accompanying amblyopia will be affected differently by operating early or late.
Even a small difference in visual function would have large socioeconomic consequences, as approximately $2 \%$ of the population undergoes strabismus surgery. The ultimate aim of the study is to reach a general guideline for ophthalmologists on surgical therapy in infantile strabismus.

\section{REFERENCES}

1. Esser J, Gieseler A, Waubke TN. Die Prognose der funktionellen Heilung des frühkindlichen Schielsyndroms. Klin Mbl Augenheilk 1981; 179:85-89.

2. Foster RS, Paul TO, Jampolsky A. Management of infantile esotropia. Am J Ophthalmol 1976; 76:291-299.

3. Ing MR. Early surgical alignment for congenital esotropia. J Pediat Ophthalmol \& Strabismus 1983; 20:11-18.

4. Ing MR. Early surgical alignment for congenital esotropia. Ophthalmology 1983; 90:132-135.

5. Leahey BD. Criteria for early surgical correction of concomitant esotropia in infants and children. Trans Am Ophthalmol Soc 1960; 58:106-117.

6. von Noorden GK. Strabismus surgery: early and very early (letter). Arch Ophthalmol 1967; 77:759.

7. von Noorden GK, Isaza A. Surgical treatment of congenital esotropia. Trans Am Acad Ophthalmol \& Otol 1972; 76:1465-1478.

8. Taylor DM. Congenital strabismus; The common sense approach. Arch Ophthalmol 1967; 77:478-484.

9. Taylor DM. Strabismus surgery: early and very early (letter). Arch Ophthalmol 1967; 77:760-761.

10. Birch EE, Stager DR, Berry P, Everett ME. Prospective assessment of acuity and stereopsis in amblyopic infantile esotropes following early surgery. Invest Ophthalmol 1990; 31:758-765.

\section{POPULATION, THERAPIES AND AIMS}

\section{Population}

The population consists of children with infantile, convergent strabismus who are aged 6 to 18 months at the time of entry into the study. The upper age limit for entry is restricted by the age at which early surgery must be completed (24th month) and by a reasonable amount of time to perform early surgery. Notwithstanding the upper age limit for entry at the 18 th month, all cases must have been documented by an orthoptist or ophthalmologist before age one, and if the child enters the study after age one, information about this previous examination must be availa- ble and summarized on the Entry Examination form. For all children with infantile, convergent strabismus aged 6 to 18 months referred to the participating clinic for the first time, an entry form must be filled out, even if they are further excluded from the study for one of the reasons given below. These data are necessary to obtain an impression of what is excluded in each participating clinic. This will be done by comparing the occurrence of exclusion criteria per clinic, and by comparing prognostic factors of patients entered into the study from patients excluded.

\section{Exclusion criteria and prognostic factors}

Children with infantile, convergent strabismus aged 6 to 18 months will be excluded from the 
study for any of the following reasons:

- Onset of strabismus after the fourth month of age. In the pilot study, it was initially suggested to use the time of onset of strabismus as a matching criterion in the main study, because a later onset indicates a better prognosis. However, $78.5 \%$ of the infants had the onset of strabismus during the first four months of life and therefore it seems wise to restrict entry into the study instead to patients who had their strabismus begin in the first four months of life. The reason for taking the first four instead of taking the first six months of life is that (a) the time of onset of strabismus is an important, prognostic parameter and we want the study groups to be as homogeneous as possible and that (b) it is generally assumed that binocular vision and stereopsis develop approximately in the fourth month of life.

- Prematurity $(<30$ weeks or $<1000$ g)

- Congenital nystagmus

- Cerebral paresis or other neurological dysfunction

- Dysmaturity

- Retardation

- Dysmorphia

- Severe motility disorders other than up- or downshoot in adduction, V-or A-pattern or limitation of abduction accompanying latent nystagmus or amblyopia

- Convergent angle $<5^{\circ}$. In this case, the angle is too small for strabismus surgery.

- : Convergent angle $>30^{\circ}$. In this case, the parents are more likely to urge for early surgery, thus creating a bias.

In rare instances, a patient can be excluded from the study for organizational reasons, if there is no chance that a patient will be able to terminate the study (e.g., foreigners) and no other participating clinic can take care of the patient.

\section{Therapies}

General guidelines for surgical procedure (established by Mr. Lee, Prof. Lennerstrand, Dr. Frosini, Dr. Faber, Dr. Prick, Dr. Kolling and Dr. Simonsz)

Surgery will consist of bilateral medial rectus recessions, combined recession-fesection surgery or Faden surgery.

Surgery will only be performed by skilled operators, i.e., consultant or senior trainee level. All cases will be seen and fully assessed two weeks post surgery.

\section{Special note on Faden surgery}

Whether or not to permit Faden surgery has been subject of intense discussion. Faden surgery is done at age $4-5$ for a completely different reason than at age 1-2: In late surgery it is done to correct convergence excess that cannot be alleviated with bifocals, whereas in early surgery, Faden surgery is done to leave the child with a constant small angle. It would seem appropriate to leave Faden surgery out altogether. However, among both early and late operating ophthalmologists there are many who consider Faden surgery an essential ingredient of their therapeutic repertoire.

Under these circumstances, there seems no other way than to leave the choice of the method of surgery to the managing ophthalmologist. Nevertheless, in both groups the cases who received Faden surgery will be compared with the other cases in their own, early or late, group to detect inconsistencies resulting from these totally different surgical approaches. 


\section{Guidelines for early surgery}

Acceptable additional surgical manoeuvres are infra- or supraplacement of the medial rectus insertions for V- or A-motility and inferior oblique weakening for marked overaction of these muscles.

Re-operation should be undertaken in cases with a residual esotropia of more than $10^{\circ}$, or in case of overcorrection. An effort should be made to complete re-operation, where undertaken, by the 24th month of age. Increase of the angle of strabismus to $10^{\circ}$ or more after age two is considered as a recurrence, and must be treated as such by renewed surgery. Re-operation may also be undertaken at a later stage if the remaining angle is between $5^{\circ}$ and $10^{\circ}$, to be determined by the managing ophthalmologist, but it must be realized that, in the outcome of the study, a re-operation will influence the angle of strabismus and the degree of binocular vision positively, but it will influence the number of operations negatively.

\section{Guidelines for late surgery}

The first operation must be carried out between 32 nd and 60 th months of age. The target of surgery should be a remaining angle of $5^{\circ}$ or less. Pronounced up- or downshoot in adduction and $\mathrm{V}$ or A-motility should be lessened.

Re-operations should be undertaken if the manifest angle of strabismus is larger than $10^{\circ}$ or in case of overcorrection. Re-operation may be undertaken if the remaining angle is between $5^{\circ}$ and $10^{\circ}$, to be determined by the managing ophthalmologist, but it must be realized that, in the outcome of the study, a re-operation will influence the angle of strabismus and the degree of binocular vision positively, but it will influence the number of operations negatively. Re-opera- tion, where undertaken, should be completed by the 66th month of age.

The last operation should be performed at least 12 months before the final evaluation at age six.

Guidelines for amblyopia treatment (established by Dr. Schulz, Prof. Roth and Dr. Simonsz)

The standardized entry examination should, whenever possible, precede the onset of treatment of amblyopia. All infants should be seen at least twice a year by the clinic that does the surgery, even when the child is treated for amblyopia by the referring ophthalmologist, to be able to intervene early when the compliance of the child or its parents is waning. Retinoscopy is performed as part of the standardized examination between 6 and 18 months of age, and at least yearly until evaluation at age six, after atropine or cyclopentolate eyedrops.

Glasses (subtracting no more than $1.0 \mathrm{D}$, preferably $0.5 \mathrm{D}$ or less, from retinoscopyincycloplegia values) are prescribed in case of:

- Spheric equivalent > 1.5 D (before early surgery this should at least have been tried)

- Anisometropia > 1 D

- Astigmatism

- after age two: $>1.5 \mathrm{D}$ or $>1 \mathrm{D}$ when oblique or against the rule

- before age two: $>2 \mathrm{D}$ or $>1.5 \mathrm{D}$ (the values are chosen a little bit higher because young infants can have large and rapidly changing astigmatism)

Bifocals are prescribed when, in cases of accommodative convergence excess, the angle of strabismus at near is reduced, and binocular vision at near is demonstrably improved (at least Bagolini positive).

Amblyopia is treated with occlusion therapy; atropine and penalization are allowed only as 
adjuncts in the treatment of amblyopia, to be determined by the managing ophthalmologist or orthoptist. How to treat amblyopia cannot be prescribed in detail, but this problem is less serious than it may seem, because there is a general consensus that the final goal of treatment of amblyopia is equal and good visual acuity of both eyes: The differences that may result from a slightly different treatment goal (for instance, patching less to disrupt fusion less) are small in comparison to the differences caused by lack of compliance of child or parents.

\section{Amblyopia treatment before early surgery (Mr. Lee)}

Cases with strongly established fixation preference and/or significant anisometropia will, after entry into the study, undergo appropriate and effective occlusion therapy to the point of nearspontaneous alternation and central fixation of the worse eye.

A corollary of adopting this strict guideline of reaching near-alternation before early surgery is that some of the cases who were entered in the study after receiving their standardized examination, with extremely severe amblyopia, will not be operated early at all. These cases will have to be inventoried individually. Adopting the guideline implies that most of us consider surgery before reaching near-alternation so unsound that it needs no further study in the trial.

The duration, kind and intensity of amblyopia treatment will be registered at each visit to the clinic. These data can be used to help explain unexpected discrepancies in visual outcome.

\section{Aims}

\section{Principal questions of the trial}

The aim of the study is to answer three principal questions concerning (1) degree of binocular vision, (2) angle of strabismus at age six and (3) treatment of amblyopia. These are considered to be the principal parameters of success of treatment at age six. The order in which the three principal questions of the trial are listed corresponds to their assigned relative importance.

\section{Is early or late surgery better, with regard to degree of binocular vision?}

For this purpose, at the final evaluation at age six, the following clinical tests will be performed:
a. Bagolini striated glasses,
b. Titmus Housefly,
c. Titmus Test circles $1-9\left(800^{\prime \prime}, 400^{\prime \prime}\right.$, $200^{\prime \prime}, 140^{\prime \prime}, 100^{\prime \prime}, 80^{\prime \prime}, 60^{\prime \prime}, 50^{\prime \prime}, 40^{\prime \prime}$ ),
d. Lang Test (old version: $1200^{\prime \prime}, 800^{\prime \prime}, 550^{\prime \prime}$ or new version: $600^{\prime \prime}, 400^{\prime \prime}, 200^{\prime \prime}$ ),
e. TNO Test plates V, VI, VII $\left(480^{\prime \prime} \& 240^{\prime \prime}\right.$, $120^{\prime \prime} \& 60^{\prime \prime}, 30^{\prime \prime} \& 15^{\prime \prime}$ ).

After the first negative response at a particular test level, the examination can be terminated. For the purpose of statistical analysis, an ordinal variable in seven levels is defined:

Level 1.1 Bagolini is negative,

1.2 only Bagolini is positive,

1.3 both Bagolini and Housefly are positive, but no Titmus circle is recognized,

1.4 at least Titmus circles $800^{\prime \prime}$ to $140 "$ are recognized,

1.5 at least Titmus circles $100^{\prime \prime}$ to $40^{\prime \prime}$ are recognized,

1.6 either all figures of the Lang Test 
or TNO Test plate V $\left(480^{\prime \prime} \&\right.$ $240^{\prime \prime}$ ) are recognized,

1.7 TNO plates VI or VII $\left(120^{\prime \prime}-\right.$ $\left.15^{\prime \prime}\right)$ are recognized.

\section{Is early or late surgery better, with regard to} manifest angle of strabismus at age six?

The angle of strabismus will be measured during fixation at distance, with full corrected glasses, with prisms and unilateral cover.

For the purpose of statistical analysis, the size of the angle is classified by defining a five-level ordinal variable. A classification based on clinical interpretation is needed because postoperative divergence carries a worse prognosis as compared to postoperative convergence.

Level 2.1 angle of squint $<-4^{\circ}$ or $\geq 15^{\circ}$,

2.2 angle $\geq-4^{\circ}$ and $<0^{\circ}$ or $\geq 10^{\circ}$ and $<15^{\circ}$,

2.3 angle $\geq 6^{\circ}$ and $<10^{\circ}$,

2.4 angle $\geq 3^{\circ}$ and $<6^{\circ}$,

2.5 angle $\geq 0^{\circ}$ and $<3^{\circ}$.

3. Is amblyopia treatment more successful against the background of early surgery than against the background of late surgery?

As a measure of the degree of amblyopia remaining after treatment, the visual acuity of the worse eye relative to that of the better eye will be taken. End point is the quotient of the visual acuities of both eyes at the final examination. This method is preferred to absolute visual acuity of the worse eye since differences in the examination procedure (such as differences in lighting or test distance) are for the larger part eliminated in this way.

Visual acuity of both eyes should be measured with $\mathrm{E}$ optotypes. Note that a line of E's is con- sidered as read successfully when three out of five $E$ 's are recognized.

Supplementary to E optotypes, Landolt circles (17.2' distance between the circles) may be used for determining the visual acuity. A line of Landolt circles is considered as read successfully when three out of five circles are recognized.

The inclusion of this third principal question has been subject to intense debate: Does the success of the amblyopia treatment have anything to do with the actual study that compares early with late strabismus surgery? Is not the success of amblyopia treatment influenced by many other factors that in no way can be controlled? These questions, posed by Mr. Lee, were good questions and one would tend to leave the issue of amblyopia out of the study entirely. This would, however, disregard the fact that an important argument employed by ophthalmologists operating late is that amblyopia treatment can be more difficult against a background of early surgery. To reconcile these contrasting lines of reasoning, the aim and methods of the study have been split into two, and the question whether early or late surgery is better for amblyopia has been rephrased to the question whether treatment of amblyopia is similarly possible against the background of early as compared to late surgery.

\section{Additional questions of the trial}

Additional questions of the trial cannot be answered in a confirmatory analysis, because the number of patients needed goes up with each additional, principal question. They will, however, be studied in an explorative fashion.

1. Number of operations and time between last operation and evaluation.

2. Remaining vertical strabismus.

3. Manifest angle of strabismus during fixa- 
tion at near, measured with full corrected glasses in primary position with prisms and unilateral cover.

4. Differences between resuits of different methods of surgery, for instance, tendencies in the results of early or late Faden surgery as compared to those of other methods.

\section{STUDY DESIGN AND PROGNOSTIC FACTORS}

\section{Study design}

Two samples are collected in a European multicentre trial. For reasons of efficiency, both samples should have approximately the same size.

For all children with infantile, convergent strabismus age 6 to 18 months referred to a participating clinic for the first time an entry form has to be filled out, even if the children are excluded from the study for any of the exclusion criteria given above.

\section{Assignment to therapy}

All participating clinics must, before entering children into the trial, choose between either early surgery (to be completed before the 25 th month) or late surgery (to be carried out between the 32nd and 60th month), for the time of their participation in the study. Hence it is not possible to operate some children early and some late and enter only one group into the trial. If a child with infantile, convergent strabismus age 6-18 months is first seen in an early operating clinic, it is and remains assigned to the early surgery group. If it is first seen in a late operating clinic, it is and remains assigned to the late surgery group.

\section{Feasibility of randomization}

During the second meeting of the Early vs. Late Infantile Strabismus Surgery Study Group in Nürnberg on June 4th, 1991, all participants agreed that, if possible, a randomized study was to be preferred. On the other hand, the difficulties that would specifically be encountered when randomizing in surgery for infantile strabismus were emphasized.

In early or late surgery for infantile strabismus, the two treatment modalities are extremely dissimilar. If the study would be randomized, one first would have to inform the parents of the patient of the possibility of surgery next week, only to postpone surgery for three years when the randomization procedure would prescribe late surgery.

Even if some very considerate parents would cooperate, the referring ophthalmologists would certainly stop sending patients. When, during the meeting, the participants were asked individually, only two of about 40 present considered a randomized study possible in their hospital. Hence, a randomized trial was considered as not feasible.

\section{Masking}

Unfortunately the two therapeutic modalities, early or late surgery, cannot be blinded. A blinded evaluation of the children at the age of six would be desirable, but is not possible since the kind of therapy varies with the evaluating clinic. In some of the participating countries, a large majority of the clinics only perform one of the two kinds of therapy.

As the trial is not randomized, the interpretability of the results largely depends on the quality of the registration. Therefore, instead of masking, the final examination will be done in 
the presence of an ophthalmologist or orthoptist from a clinic participating in the study with the other kind of therapy, i.e., during the final evaluation of the children who were operated late in a given clinic, a participant from a clinic operating early will be present, and vice versa.

\section{Prognostic factors}

During the patient recruitment phase, the distribution of known prognostic factors between the two therapy groups is monitored. To reach as much as possible homogeneity between the two therapy groups, potential skewness will be compensated by a modified recruitment procedure in case of severe lack of homogeneity.

Based on the results of a pilot-study (Early vs. Late Infantile Strabismus Surgery Study Group: How accurate is examination at age one? Proceedings of the XXth Meeting of the European Strabismological Association, Brussels, May 22-24, 1992, ed: Kaufmann H., p. 127-132), the following parameters of the entry examination have been selected as well obtainable and prognostically important: (1) spherical equivalent, (2) angle of strabismus, (3) degree of amblyopia as estimated by observing fixation behaviour and fixation in fundo (or by comparing corneal reflexes), and (4) limitation of abduction. The pilot study has shown that these parameters can be determined reliably in infants. The order in which the three parameters are listed corresponds to their assigned relative importance.

\section{Spherical equivalent}

The state of refraction is examined with retinoscopy in cycloplegia. It is measured once and retinoscopy need not be repeated if done recently. For registration on the examination form, only $1,1.5$ or 2 dioptres should be subtracted for $1 \mathrm{~m}, 0.67 \mathrm{~m}$ or $0.50 \mathrm{~m}$ retinoscopy distance; nothing else should be subtracted or added.

\section{Angle of strabismus}

The horizontal angle of strabismus is measured with prisms and corneal reflexes during fixation of an object with a light at $50 \mathrm{~cm}$ or by estimation of the location of the corneal reflexes during fixation of an object with a light at $50 \mathrm{~cm}$.

\section{Amblyopia}

In the pilot study it was shown that the degree of amblyopia could be well assessed by observing the fixation pattern, by means of direct fundoscopy with a fixation mark or by comparing corneal reflexes during monocular fixation. In addition, amblyopia could be well assessed by observing the fixation behaviour of the child with either eye. As both methods turned out to be reliable and because occasionally unlikely discrepancies were observed in the forms between the results of the two measurements, it seems wise to combine the two, for the main study, into one continuous scale encompassing the two others:

1. Poor fixation behaviour and far eccentric fixation in fundus (or determined by comparing corneal reflexes during monocular fixation).

2. Poor fixation behaviour and eccentric fixation in fundus (or determined by comparing corneal reflexes during monocular fixation).

3. Failure to maintain fixation, but central fixation in fundus (or determined by comparing corneal reflexes during monocular fixation).

4. Alternating, but preference of fixation. 
5. Cross-fixating or alternating freely.

The question which eye is dominant should be kept as an accessory because, in the pilot study, occasionally unlikely discrepancies were observed between amblyopia and dominance in the forms.

Please note that the degree of amblyopia improves when treatment is initiated. Therefore, if possible infants should be examined before starting occlusion therapy, otherwise they will be 'upgraded' to a prognostically better group.

\section{Abduction}

Limitation of abduction is measured according to the categories below:

1. Free, using pursuit movements.

2. Free, using doll's-head eye movements.

3. Passing midline but not free, using any method.

4. Not passing midline, using any method.

\section{STATISTICAL METHODS AND SAMPLE SIZE}

\section{Statistical method}

\section{Confirmatory and explorative analysis}

The three principal questions are examined in a confirmatory analysis with the intention to treat. Therefore, the error probabilities are adjusted as proposed by Bonferroni and Holm (Holm S: A simple sequentially rejective multiple test procedure. Scandinavian Journal of Statistics 1979; 6: 65-70). All other questions are analyzed with explorative methods only.

\section{Statistical analysis method}

A two-sample unpaired $t$-test is planned for the decision on the three principal questions. Heeren and d'Agostino demonstrated that this test is robust when applied to ordinal scaled data (Heeren T, d'Agostino R: Robustness of the two independent samples t-test when applied to ordinal scaled data. Statistics in Medicine 1987; 6:79-90). The validity of this method is however doubtful if the therapy groups are inhomogeneous with respect to prognostic factors. When inhomogeneity is found, the effects of prognostic factors will be included in an appropriate linear model (e.g., analysis of covariance).

\section{Dropouts and deviations from therapy}

Since the aims of the study are set according to intention to treat, every patient will be analyzed within the therapy group that the patient was originally assigned to, disregarding whether the therapy was actually carried out or not. Once patients are entered in the trial they will be examined like the other patients in the trial even when they are not operated on. Clinics with more than $5 \%$ of their patients treated differently from this protocol can be excluded from the study at the discretion of the Study Committee, comprising the Country Coordinators and the statisticians involved in the study.

In case of a dropout from the study, the parents of the child will be interviewed as to document possible reasons in detail. In case of moving house, the supervision of a patient can be transferred to another clinic.

When a patient drops out from the study definitely, it may be that results concerning the principal questions of the study are available from intermediate examinations. When these examinations took place at least one year after the last surgery, results of these could be included into the final evaluation at the discretion of the consulting biometrician. 


\section{Monitoring}

In addition to the control of prognostic factors mentioned above, aims of intermediate analyses are quality insurance, i.e., monitoring of integrity and completeness of data as well as control of the recruitment rate.

The Study Centre will prepare an annual report about the data base.

\section{Sample size determination}

\section{Method}

The sample size determination presented here is based upon the two-sample unpaired $t$-test under the assumption of homogeneous variance over both therapy groups. A simultaneous firstkind error of $5 \%$ will be accepted for the three principal criteria: binocular vision, angle of strabismus and visual acuity. The sample size determination according to Bonferroni and Holm uses $1.7 \%$ for each individual test.

The simultaneous second-kind error should not exceed $20 \%$. Interdependencies between binocular vision and angle of strabismus are surmised, whereas visual acuity is considered as being a variable independent from binocular vision and angle of strabismus. With respect to this independence, planning is based on a nominal two-kind error of $10 \%$ instead of $6.7 \%$.

\section{Assumptions for the end points}

The number of infants that have to be entered in both groups to be able to perform statistical analysis can be calculated from estimations about the approximate results that are to be expected.

\section{Binocular vision}

There are no reliable data available concerning the distribution of this principal criterion. Birch et al. (reference 9 in the introductory chapter) reported that $35 \%$ of the children operated upon early reached stereopsis with random dot stereograms. G.H. Kolling and H.J. Simonsz suggested a distribution according to the table below. The levels of binocular vision have been defined above. The number of patients that reach a higher level of binocular vision is likely to be somewhat higher, and the variance, and therefore the sample size, is more likely to be overthan to be underestimated.

level of binocular vision: $\begin{array}{lllllll}1 & 2 & 3 & 4 & 5 & 67\end{array}$ relative frequency in \%: $\begin{array}{lllllll}20 & 25 & 25 & 20 & 8 & 11\end{array}$

Given this estimation, and after assigning rank scores to the levels of binocular vision, the estimated expectance of the score is 2.8 and the variance is 1.79 (units: rank score). A difference of half a score value between the two group expectations should be recognizable. This corresponds, for instance, to an average difference between the two groups of two or three Titmus rings.

Under these assumptions, the minimal sample size required for this criterion is 200 patients per group.

\section{Angle of strabismus}

The distribution parameters of the angle of strabismus were estimated from 44 patients who were operated for infantile strabismus at the University Eye Clinic of Heidelberg. The original values were classified according to the definition of this end point described above and rank scores were assigned to the ranges of angle of strabismus defined above. Based on the data from these 44 patients, the expected value was 
estimated to be 3.3 and the variance to be 2.59 (units: rank score).

A difference of $2^{\circ}$ between the average angles of strabismus of the two groups may be considered as a minimal, clinically relevant difference in results of the two groups, equal to approximately 0.5 rank score. Based on these data, the minimal sample size is estimated to be $290 \mathrm{pa}$ tients per group.

\section{Amblyopia}

The distribution of the parameters of the visual acuity was estimated from the same series of 44 patients mentioned above. The estimates were 0.8 for the expected value of the quotient and 0.03 for the variance (units: decimal visual acuity).

A difference in visual acuity of 0.1 between the two groups may be considered as a minimal, clinically relevant difference in results of the two groups. Based on these data, the minimal sample size is estimated to be 84 patients per group.

\section{Intended sample size}

With respect to the long observation period, a high dropout rate should be anticipated. Therefore, a supplement of $30 \%$ respectively is added to the sample sizes determined above.

The maximum number of the sample sizes for the individual criteria determined above should be available for the final analysis. It is therefore intended to enroll 380 patients in both groups.

Taking into account the experiences from the pilot study, it is expected that this sample size can be realized within a period of two years.

\section{EXAMINATION AND REGISTRATION}

\section{Organization of the data registration}

The Study Centre carries out the registration of the data (address listed at the end of the protocol). Examples of all registration forms mentioned below are presented in the Appendix.

For participation in the study and after each examination of a child, a form has to be filled out immediately. The original of the filled-out form must be sent directly and immediately to the Study Centre, the copy remains at the clinic. For your convenience, questions that must always be answered have a grey-shaded box to put the answer in. Please try to answer the other questions as best as possible!

Please note also that in all forms, in the date format, the day precedes the month (ddmmyy), and not vice versa as is common in the U.S.

The Study Centre controls completeness and integrity of all forms. The quality of the registration is extremely important in a non-randomized trial. Therefore, if there are any inconsistencies or open grey-shaded boxes, the form will be sent back for correction. The Country Coordinator is informed regularly about the type and the frequency of problems that occur with registration. For ensuring the completeness of this base registration, each participating clinic agrees that the Country Coordinator could access samples from the clinic's patient registrations. Clinics can be excluded from the study if their quality of registration is too bad.

\section{Examinations and registration procedure}

\section{Examinations of an individual patient}

When a patient with convergent infantile strabismus age $6-18$ months is seen at the partici- 
pating clinic for the first time, the patient receives an entry examination, even if the patient is later excluded from the study for any reason.

It is expected that ophthalmologists who operate late will have difficulty in getting their patients early enough. The only way to resolve this problem is to ask ophthalmologists who refer patients to them, to send their strabismus patients at the age of 6 to 18 months for an initial examination and enrollment into the study, after which the patient is sent back to the referring ophthalmologist for further therapy until surgery. Note, however, that the child should also be seen twice a year by the participating clinic.

Both early and late operating participants should try to see the infants before or shortly after initiation of treatment for amblyopia, because otherwise their results will be influenced negatively, as otherwise the infants will be 'upgraded' to a prognostically better group.

If no exclusion criteria are present and after obtaining oral informed consent from one of the parents, the patient is entered into the study after receiving the entry examination. The patient is thereafter examined more briefly at sixmonth intervals.

Surgery is performed according to the guidelines in Section 3.2. Within two weeks after surgery, the patient receives a standardized postoperative examination. From this postoperative examination onwards, the intermediate examinations at six-month intervals are resumed.

Between the 6th and 7 th birthday, but at least one year after the last operation, the child receives a standardized final examination by one of the treating orthoptists or ophthalmologists, while a visiting orthoptist or ophthalmologist from a clinic participating in the study for the other operating method (early vs. late) is present at the examination (see the section about masking in Chapter 4).

\section{Entry examination}

The entry examination consists of history, preceding therapy, limitation of abduction, refraction, angle of strabismus, amblyopia, and other important parameters. These parameters must all be specifically asked for.

This is documented in the form 'Entry examination' together with one of the forms 'Intermediate examination'. In other words, the form 'Intermediate examination' is always filled out, accompanied at entry by the form 'Entry examination' (see Appendix).

Questions with open grey-shaded boxes must always be answered. In some cases, boxes not shaded grey must also be filled out. For instance, if there was previous treatment for amblyopia, this has to be described. Especially in the entry examination, any part of the examination that can be carried out, should be carried out.

\section{Recruiting patients}

If any of the exclusion criteria apply, or if the parents withhold permission, only the form 'Entry examination' accompanied by a form 'Intermediate examination' is sent to the Study Centre. In that case, a patient number is not assigned by the Study Centre.

Otherwise, the patient is entered into the study, and is assigned a patient number by the Study Centre. Please fill out a form 'Patient registration' immediately after examining the child and send it to the Study Centre, preferably by fax. The number assigned to the patient is communicated to the participating clinic with return mail.

\section{Intermediate examinations}

Intermediate examinations consist of refraction, 
angle of strabismus, amblyopia, binocular vision, and other important parameters. After the examination, the form 'Intermediate examination' (see Appendix) is filled out and sent to the Study Centre. All questions with grey-shaded boxes must always be filled out. Retinoscopy does not have to be carried out at each intermediate examination. The degree of binocular vision is assessed only in older children. If the treatment of amblyopia is altered, the changes made must be documented in detail.

\section{Operations}

An operation is documented with the form 'Operation'. The registration describes the surgical method, what muscles have been operated upon and how, and what complications have arisen. When a complication has occurred, possible influence on the postoperative result should be indicated.

\section{Final examination}

The final examination evaluates the end points concerning the principal questions of the study. All questions with a grey-shaded box must be answered in the form 'Final examination' (see Appendix).

\section{TIME SCHEDULE}

The study starts on May 1st, 1993. The time schedule from this date is as follows:

\section{Month Event}

0 : Begin of patient recruitment.

9: Closing of the list of participating clinics.

24: End of recruitment period.

42: Latest date for the first opera- tion in the early-operating group (6- month-old child recruited in month 24).

78: Latest date for the first operation in the late-operating group (6-month-old child recruited in month 24).

102: Latest date for final examination (seventh birthday of 6-month-old child recruited in month 24).

108: Latest date for corrections in the central data base.

114: Concluding report and publication of results.

The first milestone, concerning the question whether the study is feasible and possible, has just been reached: A pilot study has been carried out investigating whether children age one could be examined reliably. It is imperative that this be the case because the main study will employ a statistical analysis of the examined parameters. The pilot study showed that some parameters could be measured reliably in children age one, this speaking in favour of carrying out the main study.

The second milestone, at the end of 1995 , will concern questions about the number of children enrolled in both groups of the study, the compliance in the execution of the standardized entry examination, the compliance of the surgeons to the uniform protocol for surgery and the compliance of the ophthalmologists or orthoptists to the uniform protocol for the treatment of amblyopia.

\section{ADDITIONAL REGULATIONS}

\section{Responsibilities}

The two initiators and Study Coordinators, Dr. Kolling and Dr. Simonsz serve the study with ad- 
ministrative tasks. Statistical and methodological control as well as data entry and management of the study are carried out by the Study Centre (Zentrum zur methodischen Betreuung von Therapiestudien of the University Heidelberg, ZMBT) represented by the responsible biometrician (currently Dipl.-Inform. Med. U. Haag).

As problems of communication may rapidly ensue in an international, multilingual study, a national coordinator has been chosen for each country. Their role is to maintain contact with the participating clinics in their country. The Country Coordinators together constitute the Study Committee. The Study Committee meets regularly on request of the Study Coordinators, on request of the Study Centre, or on request of at least three of the Country Coordinators. Votes on decisions of the Study Committee are taken with simple majority.

Each participating clinic should appoint one of its staff, ophthalmologist or orthoptist, to Clinic Coordinator for the study. He or she should be familiar with the entire protocol, coordinate the study within the clinic, communicate with the Country Coordinator and with the Study Centre, make sure that all children are seen at six-months intervals and, most important, monitor that the forms are filled out correctly and completely.

\section{Participation}

Participation in the study follows after sending a Clinic Application form to the Study Centre. The Clinic Application forms can be obtained from the Country Coordinator. The clinic will receive its clinic number shortly thereafter from the Study Centre. This has to be done before entering the first child.

Clinics can enter the study until nine months after the official start of the study (see time schedule above).

\section{Changes of the study protocol and termination} of the study

Changes in this study protocol require the consent of the Study Coordinators, the Study Centre, and a majority of the Study Committee.

Such changes will be communicated to all participating clinics without delay. The study may be aborted in case of new scientific evidence making the study unnecessary, in case of severe deficiencies in the number of patients recruited, in the quality of data or in the quality of compliance.

\section{Publications}

Publications require the consent of the Study Coordinators, the Study Centre, and a majority of the Study Committee.

The results of the study will be published under the name of the study group. A footnote lists the Study Coordinators, the biometricians, the Country Coordinators and the Clinic Coordinators, so that the publication can be registered as scientific contribution by each participating clinic. The first publication concerned the pilot study mentioned above.

\section{Ethical and legal aspects}

The parents have to be informed of the purpose of the study, the pro and con arguments and the uncertainty surrounding these arguments (see Introduction). As the treatment of the infant is not altered by the study, oral informed consent is sufficient, but this has to be specifically asked for. It must be documented on the Entry Examination form. 


\section{Funding}

From November 1992 onwards, the Deutsche Forschungsgemeinschaft (DFG) supports the study with grant number Ko 1295/1-1, initially for a period of two years.

\section{Authority of the protocol}

This English version of the study protocol is the definitive guideline for the trial. If needed, it may be translated into other languages under responsibility of the Country Coordinators.

\section{COMMUNICATION}

Study Centre (please send all filled-out forms to this address):

Zentrum zur methodischen Betreuung von Therapiestudien, Mrs H. Dinkel, Universität Heidelberg, Im Neuenheimer Feld 305, W-69120 Heidelberg.

49.6221.565500. fax: 564195

Germany

Addresses of the organizers of the study:

PD Dr. H.J. Simonsz, Orthoptics \& Neuroophthalmology, Afdeling Oogheelkunde, University Hospital Dijkzigt, Dr. Molewaterplein 40, NL 3015 GD Rotterdam.

31.10.4639222, ask for beeper 3394 fax: 4635105 The Netherlands

PD Dr.med. G.H. Kolling, Schule für Orthoptik, Universitäts-Augenklinik, Im Neuenheimer Feld 400, W-69047 Heidelberg.

49.6221.566627/34/39 fax: 565422

Germany
Address of statistician of the study:

Dipl.-Inform. Med. U. Haag, Universität Heidelberg, Zentrum zur methodischen Betreuung von Therapiestudien, Im Neuenheimer Feld 305, W-69120 Heidelberg.

49.6221.564192 fax: 564195

Germany

Addresses of the Country Coordinators of the study:

Dr. A. Deák, Augenklinik, Korányi Fasor, U-6720 Szeged.

$36.62 .12321 / 10822$ fax: 22826

Hungary

Mr P. Fells, F.R.C.S., F.C. Ophth., Lower Corridor Suite, Moorfields Eye Hospital, City Road, EC1V 2PD London.

44.71.2533411 fax: 2534696 (lower corridor suite)

England

Prof. R. Frosini, Istituto di Clinica Oculista dell' Università di Firenze, Insegnamento di Ottica Fisiopatologica, Viale Morgagni 85, I-50134 Florence.

3955.411765/4379888 fax: 717953

Italy

Dr. R. Gomez de Liaño, Nuñez de Balboa 81, E-28006 Madrid.

34.1.5763229/72318

Spain

Dr. O. Haugen, Orthoptic Department, Department of Ophthalmology, University of Bergen, N-3021 Bergen.

Norway 
Dr. med. G. Klainguti, Clinique Ophtalmique, 15 Av. de France, CH-1004 Lausanne.

41.21.9637696/250211

Switzerland

Prof.Dr. K. Krzystkowa, Ophthal. Clinic Medical Acad., 38 Copernika Str, P-31501 Krakow. 4812.223272/215210/210460/214577/220411

Poland

M.C.M.E. van Lammeren, Afdeling Orthoptie, Dienst Oogziekten, U.Z. St. Rafaël, Kapucijnenvoer 33, B-3000 Leuven.

32.16.212375

Belgium

Prof. G. Lennerstrand, Institutionen för Oftalmiatrik, Huddinge sjukhus, S-14186 Huddinge. 46.8.7461000/3854/3418

Sweden

Dr.med. H. Luka, Abt. f. Schielen, Schwachsichtigkeit und Bewegungsstörungen des Auges, Allgem. öffentl. Krankenhaus der Landeshauptstadt St. Pölten, A-3100 St. Pölten.

43.512.85597/5040

43.2742.62521 (Klappe 2869)

Austria
Dr. V. Paris, Rue de Luxembourg, 48, B-6900 Marche-en-Famenne.

32.84314749

Belgium

Dr. L.J.J.M. Prick-Wenniger, Afdeling Oogheelkunde, A.M.C., Meibergdreef 9, NL 1105 AZ Amsterdam.

31.20.5663881/6715637

The Netherlands

Prof. M.A. Quéré, 19, Rue Voltaire, F-44000 Nantes.

33.40.738370/083406 fax: 084649

France

Dr. F. Reich-d'Almeida, Rua Castilho, No 65.z:Dto, P-1200 Lissabon.

351.1520619

Portugal

Dr.med. R. Weidlich, Universitäts-Augenklinik, Leninallee 8, O-4020 Halle.

37.4684.9878/81

Germany 
On this page and the following five pages the six forms used in the study are depicted. Please write to or phone the Study Centre in Heidelberg to obtain a starting set of original forms.

\section{The Early vs. Late Infantile Strabismus Surgery Study}

\section{Clinic application form}

Please use a typewriter or write clearly!

Adress of participating dinic:

Clinic coordinator:

Coordinator's assistant:

Telephone numbers:

Clinic coordinator:

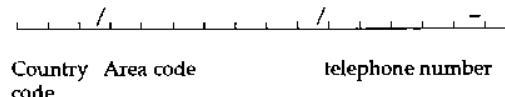

Secretary

$$
\text { code }
$$

ephone number

Coordinator's assistant:

Secretary:

Fax:

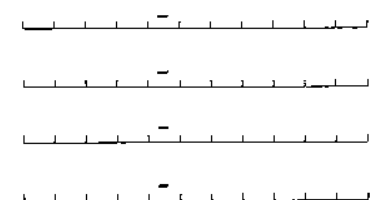

The undersigned herewith declare that, during our participation in the study for any infant with infantile, convergent strabismus age 6 to 18 months

referred to our clinic for the first time, an entry form will be sent to the Study

Centre, and when the infant is not excluded from the study it will, if necessary, be operated:

(please mark) $O$ early (before the 24th month of age)

$O$ late (between the $32 \mathrm{nd}$ and 60 th month of age)

We are familiar with the guidelines in the protocol and wish to participate in the study.

Date: Signed by Clinic Coordinator: 
The Early vs. Late Infantile Strabismus Surgery Study

\section{Patient aplication form}

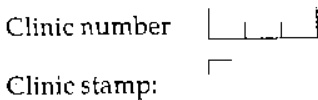

$\neg$ Patient's initials of first and last name

Date of birth (ddmmyy):

Sex:

male $O$ female

Date (ddmmyy):

Ophthalmologist / orthoptist:

We include the above mentioned patient into the study. The inclusion criteria are fulfilled and none of the exclusion criteria applies. Oral informed consent was obtained from the parents.

Signature

\begin{tabular}{|c|} 
Please send this form immediately - if possible by fax - to: \\
ZMBT Heidelberg, \\
Telefax: $49-6221$ / 56-4195 \\
or by mail, if fax is not available : \\
ZMBT Heidelberg \\
Institut für Med. Biometrie und Informatik \\
der Universität Heidelberg \\
Im Neuenheimer Feld 305 \\
W-6900 Heidelberg (from July '93 on: D-69047 Heidelberg)
\end{tabular}

To be filled out by the Study Centre:

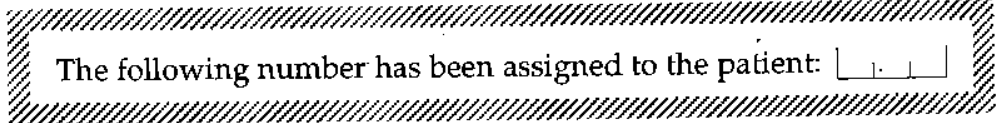


The Earty vs. Late Infantile Strabismus Surgery Study

\section{En try exam ination for m}

(must be filled out for any infant with infantile, convergent strabismus age 6 to 18 months referred to your clinic for the first time, even when excluded from the study for any reason)

Clinic number:

Clinic stamp:

Patient number: (if infant is included
into the study) Patient's initials of first and last name: Sex:

male $O$ female

Date of examination:

Examining ophthalmologist:

Examining orthoptist:

The infant complies to entry criteria: $\bigcirc$ no $\bigcirc$ yes

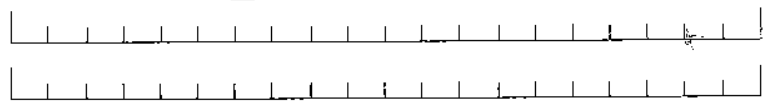

The infant complies to exclusion criteria: $\bigcirc$ no $\bigcirc$ yes: $O$ Onset of strabismus after the age of 4 months

Prematurity ( $<30$ th week or $<1000 \mathrm{~g}$ )

O Congenital nystagmus

O Cerebral palsy or other neurological deficit

Angle of strabismus $<5^{\circ}$

O Angle of strabismus $>30^{\circ}$

Oral informed consent was obtained from the parents?

$\bigcirc$ no $\bigcirc$ yes

\section{History \& previous treatment}

\begin{tabular}{|c|c|c|c|c|c|}
\hline \multicolumn{2}{|c|}{ Onset of strabismus: } & month of age & The strabismus is: & O periodic & $O$ constant \\
\hline \multirow[t]{3}{*}{ Glasses: $\quad$ No } & OYes, since $L^{\prime} L_{\perp}$ & month of age & & & \\
\hline & Glasses are & worn: & O always & O rarely & O never \\
\hline & Angle of st & rabismus with glas & sses was: 0 same & O smaller & O disappeared \\
\hline \multirow{3}{*}{$\begin{array}{l}\text { Atropin: } O \mathrm{No} \\
\text { Occlusion: } O \mathrm{No}\end{array}$} & oYes, since $L \perp$ & month of age & & & \\
\hline & OYes, since $L \perp$ & month of age & & & \\
\hline & Patch is wo & orn: & O yes & rarely & O no \\
\hline \multicolumn{6}{|c|}{ Occlusion pattern in days or in hours per day: } \\
\hline \multicolumn{2}{|l|}{ Right eye: } & days & hours/da & & \\
\hline \multicolumn{2}{|l|}{ Left eye: } & days & hours/de & & \\
\hline Both eyes open: & $\perp$ & days & & & \\
\hline \multicolumn{6}{|l|}{ Glasses worn: } \\
\hline Right: sphere: & $\sqcup \downarrow$ & cylinder: $L$ & $1,1,1$ & axis of cylinc & $11 \int_{0}$ \\
\hline Left: sphere: & 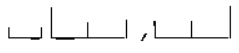 & cylinder: $1 ـ \mid$ & $\perp \perp, L \perp$ & axis of cyling & der: $\lfloor+1$ \\
\hline
\end{tabular}

Restriction of abduction is examined for the study only at entry and should be indicated below:

\begin{tabular}{|ccl}
\multicolumn{1}{c}{ Right/ Left eye } \\
0 & 0 & Free, using pursuit movements \\
0 & 0 & Free, using doll's-head eye movements \\
0 & 0 & Passing midline but not free, using any method \\
0 & 0 & Not passing midline, using any method
\end{tabular}




\section{Examination form}

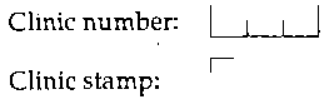

\llcorner

Examining ophthalmologist:

Examining orthoptist:

Retinoscopy:

\begin{tabular}{|c|c|c|}
\hline sphere: $ـ ا ـ ـ ـ ـ 1$ & cylinder: & axis of cylinder: $\lfloor\perp \perp\rfloor$ \\
\hline sphere: $, \perp \perp, 1, \downarrow \perp$ & cylinder: & axis of cylinder: \\
\hline New pair of glasses prescribed: $O$ No & $\begin{array}{l}\text { OYes, we prescribed the retino } \\
\text { values after subtraction }\end{array}$ & $f^{\text {copy }} L_{L, L} L_{\perp} \mathrm{d}_{\mathrm{f}}$ \\
\hline $\begin{array}{l}\text { Visual acuity with E-optotypes: } \\
\text { (decimal values preferably) }\end{array}$ & $t:\left\llcorner,\left\llcorner \_\right.\right.$or $\left\llcorner \_/ / \perp\right.$ & 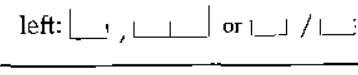 \\
\hline
\end{tabular}

Patient number:

$\neg$ Patient's initials of first and last name: Date of birth (ddmmyy):

Sex:

O male O female

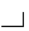

Date of examination: $\left\lfloor \_1 \quad \downarrow \perp \perp \perp\right\rfloor$

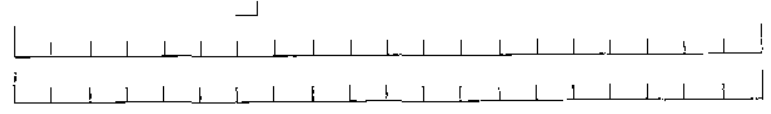

Angle of strabismus:

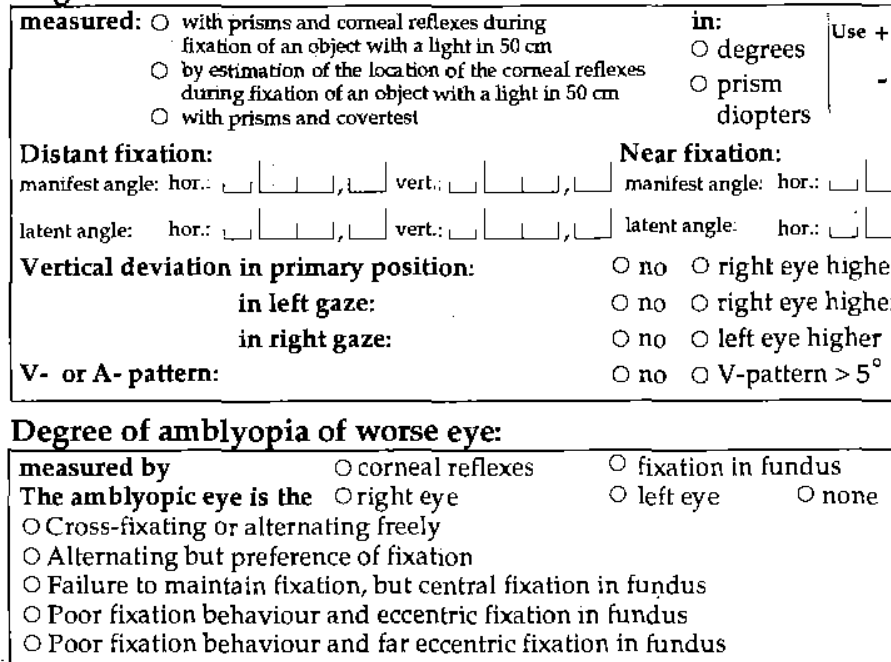

Binocular vision:

\begin{tabular}{|c|c|c|c|}
\hline Simultaneous perceptio & golini and 1 & in $50 \mathrm{~cm}$ : & Oabser \\
\hline Titmus Fly stereopsis: & O absent & O present & \\
\hline Titmus rings $800^{\prime \prime}$ to $40^{\prime \prime}$ : & O negativ & positive: & ل \\
\hline Lang-Test: & o negativ & positive & \\
\hline TNO: & O negativ & o positive: & L L \\
\hline
\end{tabular}

Latent nystagmus: $\bigcirc$ no $\circ$ yes Torticollis: $\bigcirc$ no $\bigcirc$ yes DVD: $\bigcirc$ no $\bigcirc$ yes

$\begin{aligned} & \text { At this visit amblyopia treatment } \\ & \text { was changed: }\end{aligned}$
Left eye: Both eyes open: L__ J days

The patch was worn approximately as prescribed? ONo OYes

Was the child operated during the past 6 months? ONo O Yes

Has surgery been planned for the coming 6 months? O No O Yes zMBT Heidelberg, Vers. 20, Mar. ${ }^{93}, \mathrm{Ha} / \mathrm{Di}$ 


\section{The Early vs. Late Infantile Strabismus Surgery Study}

\section{Surgery form}

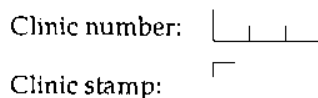

Patient number

$\neg$ Patient's initials of first and last name: $\mid$ Date of birth (ddmmyy): $|\perp|+|\perp|$ Sex:

O male $O$ female Date of examination:

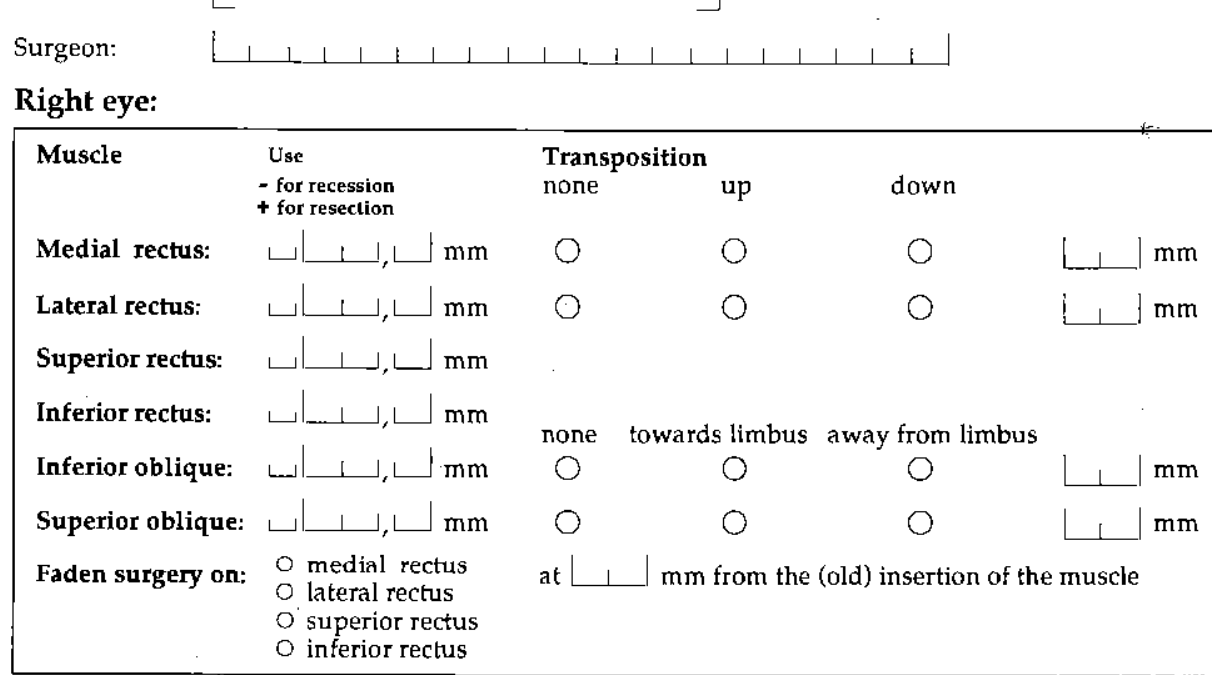

Left eye:

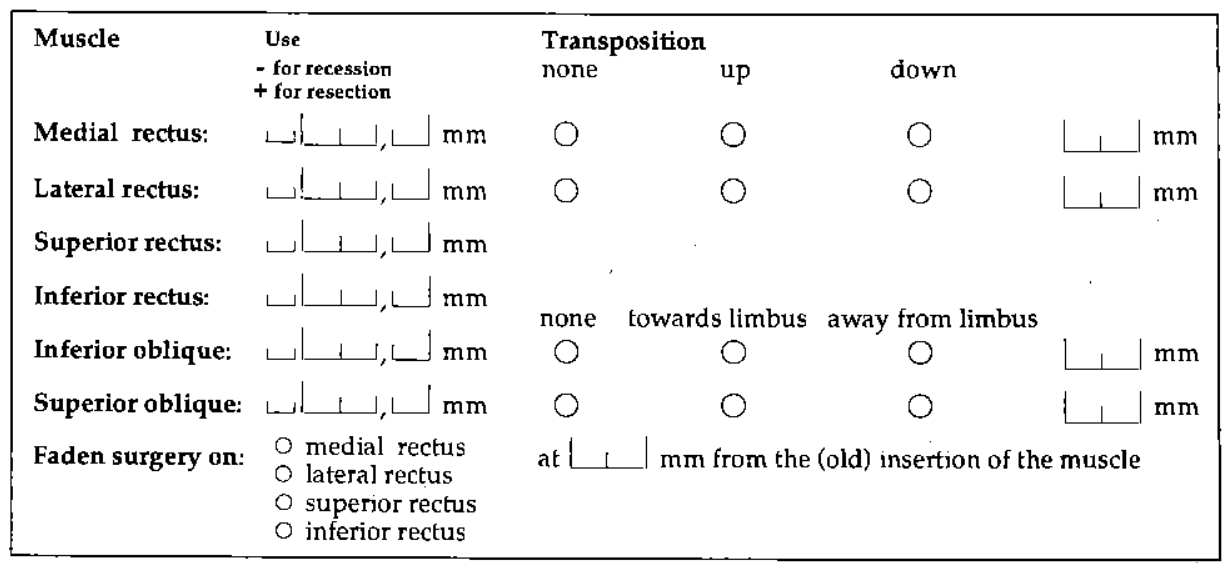

Anaesthesia problems:

Complications:

$$
\begin{aligned}
& \text { no } O \text { yes: } \\
& \text { Ono } O \text { yes: }
\end{aligned}
$$

Postoperative infections: $\bigcirc$ no $\bigcirc$ yes:

Postoperative problems: $\bigcirc$ no $\bigcirc$ yes:

Could any of the problems mentioned above influence the result of surgery? $\bigcirc$ no $\bigcirc$ yes 


\section{The Early vs. Late Infantile Strabismus Surgery Study}

\section{Fin a 1 examination form}

$\begin{array}{ll}\text { Clinic number: } & \lfloor\perp \cdot \downarrow \\ \text { Clinic stamp: } & \ulcorner\end{array}$

\llcorner

Examining ophthalmologist:

Examining orthoptist:

External observer:

from clinic number:

Number of operations:

Total number of operated muscles (1 musce operated twice counts as 2):

Last Operation on (ddmmyy):

Retinoscopy:

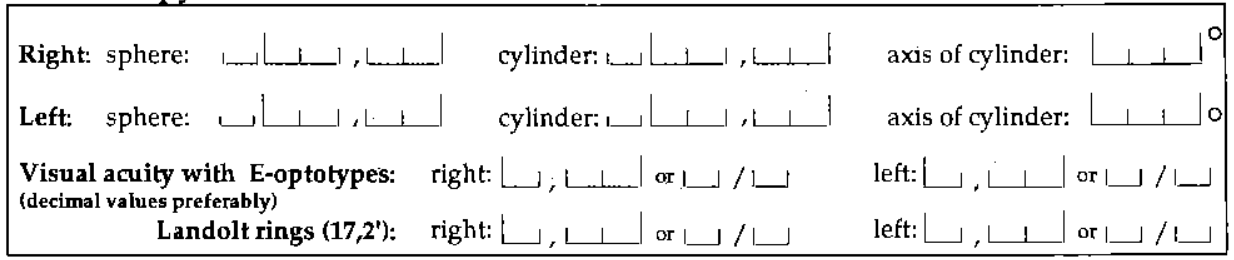

Angle of strabismus (measured with prisms and covertest):

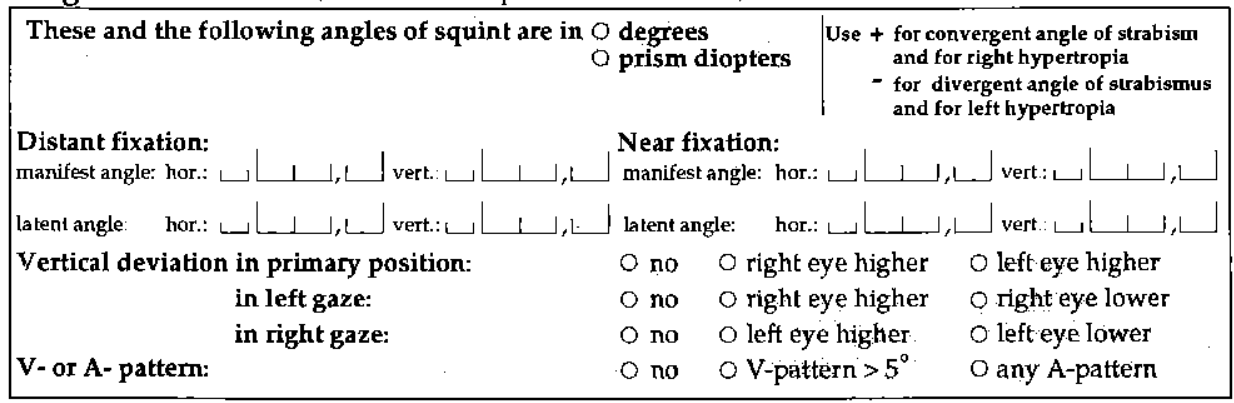

Motility:

Abduction, using pursuit movements: right eye: $O$ free $O$ not free left eye: $O$ free $O$ not free Ûp-/downshoot in adduction: vert. diverg. in left gaze: لـ Motility is otherwise: Ounrestricted O restricted, namely:

\section{Binocular vision:}

Simultaneous perception with Bagolini and light in $50 \mathrm{~cm}$ : $O$ absent $O$ present

Titmus Fly stereopsis:

Titmus rings $800^{\prime \prime}$ to $40^{\prime \prime}$ :

Lang-Test:

TNO:

$O$ absent $O$ present

$O$ negative $O$ positive: $\bigsqcup_{1} \mid \perp^{\prime \prime}$

$O$ negative $O$ positive

O negative $O$ positive: $L_{\perp} \perp \perp^{\prime \prime}$

Latent nystagmus: $O$ no $O$ yes Torticollis: $O$ no $O$ yes $\quad$ DVD: $O$ no $\bigcirc$ yes

